Pacific Journal of Mathematics

EMBEDDING OPEN 3-MANIFOLDS IN COMPACT

ALDEN H. WRIGHT 


\title{
EMBEDDING OPEN 3-MANIFOLDS IN COMPACT 3-MANIFOLDS
}

\section{Robert Messer aNd Alden Wright}

\begin{abstract}
We consider open 3-manifolds that are monotone unions of compact 3-manifolds each bounded by a torus. We give necessary and sufficient conditions for embedding such an open 3-manifold in a compact 3-manifold. We also show that if the open 3-manifold embeds in a compact 3-manifold, then it embeds in a compact 3 -manifold as the complement of the intersection of a decreasing sequence of solid tori.
\end{abstract}

1. Introduction and preliminary definitions. Kister and McMillan [5] showed that a particular contractible open 3-manifold does not embed in $S^{3}$. Haken [4] then showed that this same open 3-manifold does not embed in any compact 3-manifold. Haken's major tool was his finiteness theorem stating that there is an upper bound on the number of incompressible nonparallel surfaces in a compact 3-manifold. See [4] and §VI of [12]. This theorem is also the major tool in this paper.

The spaces and maps that we will consider are intended to be in the piecewise linear category. An n-manifold is a separable metric space such that each point has a neighborhood homeomorphic to an $n$-cell. A submanifold of an $n$-manifold is a subspace that is also an $n$-manifold. A $k$-manifold $F$ is properly embedded in an $n$-manifold $N$ if and only if $F$ is a closed subset of $N$ and $F \cap$ $\partial N=\partial F$. A surface is a connected 2-manifold; a planar surface is a surface that can be embedded in a disk. A punctured disk is a planar surface obtained by removing from the interior of a disk the interiors of a finite collection of disjoint subdisks. Similarly a punctured 3 -cell is a compact 3-manifold obtained by removing from the interior of a 3-cell the interiors of a finite collection of disjoint 3-cells.

Let $I$ denote the unit interval $[0,1]$; let $I_{i}$ denote the interval $[-i, i]$.

Two properly embedded surfaces $F$ and $F^{\prime}$ are parallel in a 3manifold $N$ if and only if there is an embedding of $(F \times I, \partial F \times I)$ into $(N, \partial N)$ such that $F$ is the image of $F \times\{0\}$ and $F^{\prime}$ is the image of $F \times\{1\}$. A collection of properly embedded surfaces is parallel if and only if any two disjoint surfaces in the collection are parallel. The corresponding definitions for parallel simple closed curves in a 2-manifold are similar.

A 2-manifold $F$ properly embedded in a 3-manifold $N$ is compressible if and only if either $F$ is a 2 -sphere that bounds a 3-cell in 
$N$, or there is a compressing disk for $F$, that is, a disk $D$ in $N$ such that $D \cap \partial F=\partial D$ and $\partial D$ is a nontrivial curve on $F$. Otherwise $F$ is incompressible.

An open 3-manifold $N$ is simply connected at infinity if and only if each compact subset of $N$ is contained in a compact submanifold $B$ of $N$ such that $N-B$ is simply connected.

In this paper $M$ will denote an open 3-manifold such that $M=$ $\bigcup_{i=0}^{\infty} M_{i}$ where each $M_{i}$ is a compact, connected 3-manifold with a single, torus boundary component, and $M_{i} \subseteq \operatorname{Int}\left(M_{i+1}\right)$. For $i>0$ let $K_{i}=M_{i}-\operatorname{Int}\left(M_{i-1}\right)$.

2. Conditions for embedding $M$ in a compact 3-manifold. Theorems 2.3 and 2.4 provide a necessary and sufficient condition for $M$ to embed in a compact 3-manifold. The following two preliminary lemmas will be used in the proof of Theorem 2.3.

Lemma 2.1. Suppose $E$ and $F$ are tori and $E$ is embedded in Int $(F \times I)$ so that it separates the components of $\partial(F \times I)$. Then $E$ is parallel to $\partial(F \times I)$.

Proof. Notice that $E$ must be incompressible in $F \times I$. Otherwise we could use a compressing disk for $E$ to obtain a 2-sphere in $F \times I$ that separates the components of $\partial(F \times I)$. This contradicts the fact that $F \times I$ is irreducible. The conclusion now follows by Haken's lemma on page 91 of [4].

Lemma 2.2. Suppose $M$ is embedded in a compact 3-manifold $N$. Suppose there is an integer $j$ such that for $i>j, \partial M_{i-1}$ and $\partial M_{i}$ are nonparallel and incompressible in $K_{i}$. Then for some $n \geqq j$ there is a compressing disk $D$ for $\partial M_{n}$ in $N-\operatorname{Int}\left(M_{n}\right)$. Furthermore, if any such disk $D$ and an open regular neighborhood of $D$ in $N-\operatorname{Int}\left(M_{n}\right)$ satisfy the following three properties for $n<i \leqq k$, then $D$ and the open regular neighborhood can be modified in $N$ Int $\left(M_{k}\right)$ so that these properties hold for $n<i \leqq k+1$.

(1) The components of $D \cap K_{i}$ are punctured disks that are parallel and properly embedded in $K_{i}$ and each has exactly one boundary component on $\partial M_{i-1}$.

(2) The components of $D \cap\left(N-\operatorname{Int}\left(M_{i}\right)\right)$ are compressing disks for $\partial M_{i}$ in $N-\operatorname{Int}\left(M_{i}\right)$.

(3) The open regular neighborhood of $D$ in $N-\operatorname{Int}\left(M_{n}\right)$ is homeomorphic to $D \times \boldsymbol{R}$ under a homeomorphism that maps the intersection with $\partial M_{i}$ onto $\left(D \cap \partial M_{i}\right) \times \boldsymbol{R}$ and the intersection with $K_{i}$ onto $\left(D \cap K_{i}\right) \times \boldsymbol{R}$. 
Proof. Consider the infinite collection of surfaces $\partial M_{i}$ for $i \geqq j$ in the compact 3-manifold $N-\operatorname{Int}\left(M_{j}\right)$. If any two of these surfaces were parallel, Lemma 2.1 would imply that all of the intermediate surfaces would be parallel. This is contrary to the hypothesis that no two adjacent surfaces are parallel. Haken's finiteness theorem implies that there is a compressing disk $D$ for one of the surfaces $\partial M_{n}$. If $D$ were contained in $M$ - Int $\left(M_{j}\right)$, it would intersect at most a finite number of the $\partial M_{i}$. We could put it in general position with respect to these surfaces so that the intersection would be a finite collection of simple closed curves. Then we could modify $D$ to eliminate the curves of intersection that were trivial on the surfaces. Of the remaining curves, one that was innermost on $D$ would bound a subdisk of $D$ that is contained in some $K_{i}$. This is contrary to the hypothesis that $\partial M_{i-1}$ and $\partial M_{i}$ are incompressible in $K_{i}$. Thus $D$ is properly embedded in $N-\operatorname{Int}\left(M_{n}\right)$.

Suppose there is an integer $k \geqq n$ such that $D$ and an open regular neighborhood of $D$ in $N$ - Int $\left(M_{n}\right)$ satisfy the three properties for $n<i \leqq k$. We can modify $D$ in $N-\operatorname{Int}\left(M_{k}\right)$ to eliminate any simple closed curves of intersection between $D$ and $\partial M_{k+1}$ that are trivial on $\partial M_{k+1}$. The intersection $D \cap \partial M_{k+1}$ must still be nonempty since $\partial M_{k}$ is incompressible in $K_{k+1}$ and the curves of $D \cap \partial M_{k}$ are nontrivial on $\partial M_{k}$. Of the curves of $D \cap \partial M_{k+1}$, choose one that is innermost on $D$ and consider the subdisk $E$ of $D$ that it bounds. Since $\partial M_{k+1}$ is incompressible in $K_{k+1}, E$ must be properly embedded in $N-\operatorname{Int}\left(M_{k+1}\right)$. Also choose one of the components of $D \cap K_{k+1}$. By property (2), this component is a punctured disk disk with exactly one boundary component on $\partial M_{k}$. Because of the above modifications of $D$, the remaining boundary curves of this punctured form a collection of parallel simple closed curves that are nontrivial on $\partial M_{k+1}$. For each of the curves of $D \cap \partial M_{k}$, modify $D$ in $K_{k+1}$ by attaching copies of this punctured disk that are parallel in $K_{k+1}$. Then to each of the resulting curves of intersection with $\partial M_{k+1}$, attach parallel copies of $E$ in $N$ - Int $\left(M_{k+1}\right)$.

Finally choose an open regular neighborhood of $D \cap\left(N-\operatorname{Int}\left(M_{k}\right)\right)$ in $N$ - Int $\left(M_{k}\right)$ such that its intersection with $\partial M_{k+1}$ is an open regular neighborhood of $D \cap \partial M_{k+1}$ in $\partial M_{k+1}$, its intersection with $K_{k+1}$ is an open regular neighborhood of $D \cap K_{k+1}$ in $K_{k+1}$, and its intersection with $\partial M_{k}$ agrees with the intersection of $\partial M_{k}$ with the original open regular neighborhood of $D$. Then the original open regular neighborbood in $M_{k}$ and the new open regular neighborhood in $N$ - Int $\left(M_{k}\right)$ can be combined to form the open regular neighborhood satisfying property (3).

THEOREM 2.3. Suppose $M$ can be embedded in a compact 3- 
manifold. Then either $M$ is simply connected at infinity and contains no infinite collection of disjoint fake 3-cells, or there is an integer $n$ such that for each $i>n$ there is a punctured dist $P_{i}$ properly embedded in $K_{i}$ with the following properties.

(1) $P_{i} \cap \partial M_{r-1}$ is a simple closed curve that is nontrivial on $\partial M_{i-1}$, and $P_{i} \cap \partial M_{i}$ is a nonempty collection of simple closed curves that are parallel and nontrivial on $\partial M_{i}$.

(2) When a solid torus is attached to $K_{i}$ along $\partial M_{i}$ so that the components of $P_{i} \cap \partial M_{i}$ are meridional curves of the solid torus, the resulting 3-manifold is a solid torus.

(3) For $i>n+1$, the simple closed curve $P_{i} \cap \partial M_{\imath-1}$ is parallel to the curves of $P_{\imath-1} \cap \partial M_{i-1}$ on $\partial M_{i-1}$.

Proof. Suppose $M$ is embedded in a compact 3 -manifold $N$. If an infinite number of the surfaces $\partial M_{i}$ are parallel in $N-\operatorname{Int}\left(M_{0}\right)$, Lemma 2.1 implies that we can choose $n$ so that all the surfaces $\partial M_{i}$ for $i \geqq n$ are parallel. Then we can choose vertical annuli $P_{i}$ in $K_{i} \cong \partial M_{i} \times I$ for $i>n$ that satisfy the three properties.

If this is not the case, then for any $i$ there is a $k \geqq i$ such that $\partial M_{i}$ is paralle! to $\partial M_{k}$ but not parallel to $\partial M_{k+j}$ for $j \geqq 1$. Lemma 2.1 implies that $\partial M_{i}, \partial M_{i+1}, \cdots, \partial M_{k}$ are parallel. Thus, a punctured disk properly embedded in $M_{k}$ - Int $\left(M_{\imath}\right)$ that satisfies the three properties can be adjusted so that the components of its intersection with $M_{i+1}-\operatorname{Int}\left(M_{i}\right), \cdots, M_{k}-\operatorname{Int}\left(M_{k-1}\right)$ satisfy the three properties. Therefore by choosing a subsequence of the surfaces $\partial M_{i}$ and reindexing, we can assume no two of these surfaces are parallel.

If there is an infinite number of values of $i$ such that $\partial M_{i}$ is compressible in $K_{i} \cup K_{i+1}$, we can find either an infinite sequence of even values of $i$ or an infinite sequence of odd values of $i$ with this property. In either case we can modify each of the corresponding submanifolds $M_{i}$ by attaching a 2-handle in $K_{i+1}$ to $M_{i}$ or cutting $M_{i}$ along a 1-handle in $K_{i}$. Notice that if $M_{i}$ is modified, then $M_{i-1}$ and $M_{i+1}$ are unchanged. It follows that $M$ can be written as the union of a nested sequence of compact, connected submanifolds each having a single, 2 -sphere boundary component. Of the complementary domains of $N$ determined by the union of these 2-spheres, Kneser's theorem [6] implies that all but a finite number must be homeomorphic to $S^{2} \times I$. It thus follows that $M$ is simply connected at infinity and contains no infinite collection of disjoint fake 3-cells.

Now we need to consider only the case that there is an integer $j$ such that none of the tori $\partial M_{i}$ for $i \geqq j$ is parallel in $N-\operatorname{Int}\left(M_{0}\right)$ to any other, and such that for each $i>j, \partial M_{i-1}$ and $\partial M_{i}$ are incompressible in $K_{\imath}$ 。 By Lemma 2.2 there is an integer $n \geqq j$ and a compressing disk $D$ for $\partial M_{n}$ in $N-\operatorname{Int}\left(M_{n}\right)$. For any $k>n$ we can 
inductively modify $D$ and the open regular neighborhood of $D$ so that the three properties of Lemma 2.2 are satisfied for $n<i \leqq k$. Let $P_{i}$ be a component of $D \cap K_{i}$ and let $D_{i}$ be the component of $D \cap\left(N-\operatorname{Int}\left(M_{i}\right)\right)$ that contains $P_{i+1}$. Notice that as long as $k$ is chosen to be greater than $i$, the punctured disks $P_{i}$ do not depend on the value of $k$. Notice also that the $P_{i}$ satisfy properties (1) and (3) of the conclusion of this theorem.

Let us identify the open regular neighborhood of $D$ in $N-$ Int $\left(M_{n}\right)$ with $D \times R$ by a homeomorphism as indicated in property (3) of Lemma 2.2. Let

$$
K_{i}^{*}=\left(M_{i} \cup D_{i-1} \times I_{i}\right)-\operatorname{Int}\left(M_{i-1} \cup D_{i-1} \times I_{i-1}\right) .
$$

By adding the orientation preserving 1-handle $D_{i-1} \times I_{i-1}$ to $K_{i}^{*}$, we obtain $\left(M_{i} \cup D_{i-1} \times I_{i}\right)$ - Int $\left(M_{i-1}\right)$. By attaching 3-cells to the 2sphere boundary components of $\left(M_{i} \cup D_{i-1} \times I_{i}\right)$ - Int $\left(M_{i-1}\right)$, we obtain the 3-manifold described in property (2) of the conclusion of this theorem. Hence this 3 -manifold is a solid torus if and only if $K_{i}^{*}$ is a punctured 3-cell.

In any case $K_{i}^{*}$ is bounded by 2 -spheres. So any $K_{i}^{*}$ that is not a punctured 3-cell contributes at least one nontrivial factor to a connected sum decomposition of the compact 3-manifold $N$. By Kneser's theorem [6] there can only be a finite number of such values of $i$. Therefore we can choose an integer $n$ so that property (2) is also satisfied for all $i>n$.

THEOREM 2.4. Suppose there is an integer $n$ such that for $i>n$ there is a punctured disk $P_{i}$ properly embedded in $K_{i}$ satisfying the three properties of the conclusion of Theorem 2.3. Then $M$ can be embedded in a compact 3-manifold $N$ so that $N$ - Int $\left(M_{i}\right)$ is a solid torus for all $i \geqq n$.

REMARK. If $M$ is simply connected at infinity and contains no infinite collection of disjoint fake 3-cells, then, by the techniques of Edwards [3], the one point compactification of $M$ is a compact 3manifold. Together with this fact, Theorem 2.4 thus provides the converse to Theorem 2.3.

Proof of Theorem 2.4. We want to use the punctured disks $P_{i}$ to construct a planar surface $P$ that has a single boundary component and is properly embedded in $M-\operatorname{Int}\left(M_{n}\right)$. The planar surface $P$ can be constructed inductively so that it satisfies the following defining properties. First $P \cap K_{n+1}=P_{n+1}$. Second, for $i>n+1, \quad P \cap K_{i}$ consists of copies of $P_{i}$ that are properly embedded and parallel in 
$K_{i}$ and are such that the boundary curves on $\partial M_{i-1}$ coincide with the boundary curves of $P \cap M_{i-1}$ on $\partial M_{i-1}$.

Next we want to embed $M$ in an open 3 -manifold $M^{*}$ so that $P$ is contained in a disk $D$ in $M^{*}$. We can choose a regular neighborhood of $P$ in $M$ - Int $\left(M_{n}\right)$ that intersects each $\partial M_{i}$ in a regular neighborhood of $P \cap \partial M_{i}$ in $\partial M_{i}$. The topological interior of this regular neighborhood in $M-\operatorname{Int}\left(M_{n}\right)$ is an open regular neighborhood of $P$ in $M$ - Int $\left(M_{n}\right)$. We can identify this open regular neighborhood with $P \times \boldsymbol{R}$ by a homeomorphism that identifies the intersection of the open neighborhood and $\partial M_{i}$ with $\left(P \cap \partial M_{i}\right) \times \boldsymbol{R}$.

We can embed $P$ as a subset of a disk $D$ so that $\partial D$ is the image of $\partial P$. Then for each $i \geqq n, P \cap \partial M_{\imath}$ determines a collection of simple closed curves on $D$ each bounding a subdisk of $D$ that contains a component of $P \cap\left(M-\operatorname{Int}\left(M_{i}\right)\right)$. Let $D_{i}$ be the union of these subdisks. Let $M_{i}^{*}$ be the compact 3-manifold obtained by attaching to a copy of $M_{i}$ a collection of 2-handles $D_{\imath} \times I_{\imath}$ along $\partial M_{i}$. Choose the attaching maps so that the product structure of $\partial D_{i} \times I_{i}$ agrees with the product structure of $\left(P \cap \partial M_{i}\right) \times I_{i}$. Notice that for $i>n$, there is an embedding of $M_{i-1}^{*}$ into $M_{i}^{*}$ determined by the embedding of $M_{i-1}$ in $M_{i}$ and the standard embedding of $D_{i} \times I_{\imath-1}$ into $D_{i} \times I_{i}$. Let $K_{i}^{*}=M_{i}^{*}-\operatorname{Int}\left(M_{i-1}^{*}\right)$. Now let $M^{*}$ be the open 3-manifold obtained from $M_{n}^{*}$ by inductively attaching the $K_{i}^{*}$ so that

$$
M^{*}=M_{n}^{*} \cup\left(\bigcup_{i=n+1}^{\infty} K_{i}^{*}\right)=\bigcup_{i=n}^{\infty} M_{i}^{*}
$$

Since $P$ can be embedded in $D$ and each $M_{i}$ can be embedded in the corresponding $M_{i}^{*}$, this will give the desired embeddings of $M$ and $P$ into $M^{*}$.

By removing the interiors of a collection of disjoint 3-cells from the solid torus described in property (2) of Theorem 2.3, we can obtain $M_{i}^{*}-\operatorname{Int}\left(M_{i-1}\right)$. Furthermore, by cutting $M_{i}^{*}-\operatorname{Int}\left(M_{\imath_{-1}}\right)$ along the 1 -handles $D_{i-1} \times I_{i-1}$ we can obtain

$$
K_{i}^{*}=M_{i}^{*}-\operatorname{Int}\left(M_{i-1}^{*}\right)=M_{i}^{*}-\operatorname{Int}\left(M_{i-1} \cup D_{i-1} \times I_{i-1}\right) \text {. }
$$

Thus, $K_{i}^{*}$ is the union of a collection of punctured 3-cells. Notice that each component of $K_{i}^{*}$ intersects $M_{i-1}^{*}$ in a single 2-sphere.

Let $N$ be the compact 3-manifold obtained by attaching a solid torus to a copy of $M_{n}$ along $\partial M_{n}$ so that $\partial D$ is a meridional curve of the solid torus. Then we can extend the embedding of $M_{n}$ into $N$ to an embedding of $M_{n}^{*}=M_{n} \cup D \times I_{n}$ into $N$. Finally, we can inductively embed the punctured 3-cells of $K_{n+1}^{*}, K_{n+2}^{*}, \cdots$ in the remaining 3-cells of the solid torus. This gives the desired embedding 
of $M^{*}$, and hence also of $M$, into $N$. It follows from the construction that $N-\operatorname{Int}\left(M_{i}\right)$ is a solid torus for all $i \geqq n$.

CoRollary 2.5. Suppose $M$ can be embedded in a compact 3manifold. Then there is an integer $n$ such that $M-\operatorname{Int}\left(M_{n}\right)$ can be embedded in a solid torus as the complement of the intersection of a decreasing sequence of solid tori. In particular, $M$ is orientable at infinity.

Proof. By Theorem 2.3, $M$ is either simply connected at infinity and contains no infinite collection of disjoint fake 3-cells, or $M$ contains the punctured disks satisfying the three properties. In the first case, the conclusion follows by the remark made after the statement of Theorem 2.4. Otherwise we can apply Theorem 2.4.

REMARK. The referee has pointed out that this corollary may be proved by using the main result of a paper [7] by Knoblauch. If $M$ is embedded in a compact, orientable 3-manifold, Knoblauch's result implies that $M-\operatorname{Int}\left(M_{j}\right)$ can be embedded in $S^{3}$ for some $j$. As in the proof of Theorem 2.3, we can assume that for $i \geqq j$ none of the tori $\partial M_{i}$ is parallel in $M-\operatorname{Int}\left(M_{j}\right)$ to any other, and that for each $i>j, \partial M_{i-1}$ and $\partial M_{i}$ are incompressible in $K_{i}$; otherwise the result of the corollary follows easily. Now with $M-\operatorname{Int}\left(M_{j}\right)$ embedded in $S^{3}$ we can use Haken's theorem to find an integer $n$ so that if $i>n, \partial M_{i}$ bounds a solid torus containing $M-\operatorname{Int}\left(M_{i}\right)$.

On the other hand, suppose that $M$ is embedded in a compact, nonorientable 3-manifold $N$. For some $n$ we must have that $M$ Int $\left(M_{n}\right)$ is orientable. Otherwise for some integer $j$ we could find an orientation reversing loop $\alpha$ in $\operatorname{Int}\left(M_{j}\right)$ and an orientation reversing loop $\beta$ in $M-M_{j}$ so that $\alpha$ and $\beta$ represent the same element of the finite group $H_{1}\left(N ; Z_{2}\right)$. A Mayer-Vietoris sequence argument would give a loop $\gamma$ in $\partial M_{j}$ that represents this same element of $H_{1}\left(N ; Z_{2}\right)$. But $\partial M_{j}$ is orientable and 2 -sided, so $\gamma$ would be orientation preserving. Now since $M-\operatorname{Int}\left(M_{n}\right)$ is orientable, it embeds in the orientable double cover of $N$, and we can apply the argument of the previous paragraph.

3. The existence of the punctured disks. In this section we will consider conditions on $K_{i}=M_{i}-\operatorname{Int}\left(M_{i-1}\right)$ that are necessary for the existence of a properly embedded punctured disk satisfying properties (1) and (2) of Theorem 2.3.

The following notation and conventions will be used throughout this section. All homology groups will be with coefficients in $Z$. If $c$ is a collection of oriented simple closed curves in an $n$-manifold, 
let [c] denote the sum of the elements of the first homology group of the $n$-manifold represented by the curves of $c$. Let us assume there is a homeomorphism $h: K_{i} \rightarrow L$ where $L$ is the complement of the interiors of two disjoint solid tori $T$ and $T^{\prime \prime}$ in a homology 3sphere $S$. In Corollary 2.5 we have shown that if $M$ embeds in a compact 3-manifold, then this assumption holds for all but a finite number of values of $i$. Let us also assume that $h\left(\partial M_{i}\right)=\partial T$ and $h\left(\partial M_{i-1}\right)=\partial T^{\prime}$. If $c$ is a union of simple closed curves in $S$ and $d$ is a simple closed curve disjoint from $c$, then the linking number of $c$ with $d$ is the absolute value of the multiple of a generator determined by $[c]$ in $H_{1}(S-d) \cong Z$. Let $q$ denote the linking number of the centerline of $T$ with the centerline of $T^{\prime}$. It can be shown that $q$ is independent of the choice of $h, L$, and $S$. Choose transverse oriented simple closed curves $l$ and $m$ on $\partial T$ so that $l$ bounds a surface in $S-\operatorname{Int}(T)$ and $m$ bounds a disk in $T$. A simple closed curve on $\partial T$ that represents $\pm \alpha[l] \pm \beta[m] \neq 0$ in $H_{1}(\partial T)$ for $\alpha \geqq 0$ and $\beta \geqq 0$ will be called an $(\alpha, \beta)$-curve on $\partial T$. It can be shown that $\alpha$ and $\beta$ are relatively prime. On $\partial T^{\prime}$ choose $l^{\prime}$ and $m^{\prime}$ similarly. The corresponding definition of an $(\alpha, \beta)$-curve on $\partial T^{\prime}$ is also similar.

THEOREM 3.1. Let $P$ be a punctured disk properly embedded in $L$ with the following properties.

(1) $P \cap \partial T^{\prime}$ is an $(\alpha, \beta)$-curve on $\partial T^{\prime \prime}$, and $P \cap \partial T$ is a nonempty collection of simple closed curves that are parallel and nontrivial on $\partial T$.

(2) When a solid torus $T^{*}$ is attached to $L$ along $\partial T$ so that the components of $P \cap \partial T$ are meridional curves of $T^{*}, L \cup T^{*}$ is a solid torus.

Give the components of $\partial P$ the orientations induced from an orientation chosen for $P$. If $q=0$, then

(a) $\alpha=1$ and $\beta=0$,

(b) $[P \cap \partial T]=0$ in $H_{1}(\partial T)$, and

(c) each component of $P \cap \partial T$ is a $(\gamma, 1)$-curve on $\partial T$ for some integer $\gamma$.

If $q>0$, then

(d) $\beta$ is divisible by $q^{2}$,

(e) $[P \cap \partial T]= \pm(\beta / q)[l] \pm \alpha q[m]$ in $H_{1}(\partial T)$,

(f) each component of $P \cap \partial T$ is a $\left(\beta / q^{2}, \alpha\right)$-curve on $\partial T$, and

(g) if $q>1$, then $\alpha \neq 0$.

REMark. Properties (1) and (2) of this theorem are restatements of the corresponding properties of Theorem 2.3. 
Proof of Theorem 3.1. Let us first consider the case that $q=0$. Then $P \cap \partial T$ does not link the centerline of $T^{\prime \prime}$, so neither does $P \cap \partial T^{\prime}$. This implies that $\beta=0$, which in turn implies that $\alpha=1$. Since we can extend $P$ to a meridional disk of the solid torus $L \cup T^{*}$, it follows that $T^{\prime} \cup L \cup T^{*}$ is homeomorphic to the 3 -sphere $S^{3}$. In particular $H_{1}\left(T^{\prime} \cup L \cup T^{*}\right) \cong(0)$. Since $T^{\prime} \cup L$ is the complement of Int $(T)$ in the homology 3-sphere $S, H_{1}\left(T^{\prime} \cup L\right) \cong Z$ and is generated by $[m]$. Thus a meridional disk of $T^{*}$ must have been attached to a $(\gamma, 1)$-curve on $\partial T$ for some integer $\gamma$. Since any such curve links the centerline of $T$, but $P \cap \partial T^{\prime}$ does not, we must have $[P \cap \partial T]=0$ in $H_{1}(\partial T)$.

Let us now consider the case that $q>0$. As in the first case, $T^{\prime} \cup L \cup T^{*}$ is homeomorphic to $S^{2} \times S^{1}$ if $\alpha=0, S^{3}$ if $\alpha=1$, or to the lens space $L_{\alpha, \beta}$ if $\alpha>1$. In particular $H_{1}\left(T^{\prime} \cup L \cup T^{*}\right) \cong Z / \alpha Z$.

Also recall that $H_{1}\left(T^{\prime} \cup L\right) \cong Z$ and is generated by $[m]$. Thus we can also compute $H_{1}\left(T^{\prime} \cup L \cup T^{*}\right)$ by considering the way a meridional disk of $T^{*}$ is attached to $T^{\prime} \cup L$. Now the oriented simple closed curve $P \cap \partial T^{\prime}$ has linking number $\alpha q$ with the centerline of $T$ and $\beta$ with the centerline of $T^{\prime}$. Since the oriented curves $P \cap \partial T$ are homologous to $P \cap \partial T^{\prime}, P \cap \partial T$ also has linking number $\alpha q$ with the centerline of $T$ and $\beta$ with the centerline of $T^{\prime}$. Since each factor of $[l]$ in an element of $H_{1}(\partial T)$ corresponds to linking the centerline of $T^{\prime}$ a total of $q$ times, it follows that $[P \cap \partial T]=$ $\pm(\beta / q)[l] \pm \alpha q[m]$ in $H_{1}(\partial T)$. Thus each component of $P \cap \partial T$ is a $(\beta / d q, \alpha q / d)$-curve where $d$ is the greatest common divisor $\beta / q$ and $\alpha q$. Therefore $H_{1}\left(T^{\prime} \cup L \cup T^{*}\right) \cong Z /(\alpha q / d) Z$, and so $\alpha q / d=\alpha$. Now if $\alpha \neq 0$, then $q=d$. If $\alpha=0$, then $\beta=1$, so we must have $q=1$. In either case each component of $P \cap \partial T$ is a $\left(\beta / q^{2}, \alpha\right)$-curve.

4. Applications and examples. In the previous section we investigated the existence of a punctured disk properly embedded in $K_{i}$ satisfying properties (1) and (2) of Theorem 2.3. In this section we will give conditions that also involve the matching of the boundary curves of the punctured disk at one stage with the boundary curves of the punctured disk at the previous stage, that is condition (3) of Theorem 2.3. We will also consider several examples as typical applications of these results.

Throughout this section we will assume there in an integer $n$ such that for each $i>n$ there is a homeomorphism $h_{i}: K_{i} \rightarrow L_{i}$ where $L_{i}$ is the complement of the interiors of two disjoint solid tori $T_{i}$ and $T^{\prime}{ }_{i}$ in the 3 -sphere $S^{3}$. As in $\S 3$ we assume $h_{i}\left(\partial M_{i}\right)=\partial T_{i}$ and $h_{i}\left(\partial M_{i-1}\right)=\partial T_{i}^{\prime}$. We also define the linking number $q_{i}$ and choose the longitudinal and meridional curves $l_{i}, m_{i}, l_{i}^{\prime}, m_{i}^{\prime}$ similarly. For each $i>n$ let 


$$
g_{i}=\left(h_{i} \mid \partial M_{i}\right) \circ\left(h_{i+1}^{-1} \mid \partial T_{i+1}^{\prime}\right): \partial T_{i+1}^{\prime} \longrightarrow \partial T_{i} .
$$

Then $g_{i}$ describes the matching between $L_{i+1}$ and $L_{i}$.

A solid torus $T$ is unknotted in a closed 3-manifold if and only if there is a compressing disk $D$ for $\partial T$ such that $\partial D$ is transverse to a meridional curve of $T$. The wrapping number of a solid torus $T^{\prime}$ in the interior of a solid torus $T$ is the minimum number of components in the intersection between $T^{\prime}$ and a meridional disk of $T$.

THEOREM 4.1. Suppose each $q_{i}=0$. Let $Q_{i}$ be the 3-manifold obtained when a solid torus is attached to $T_{i}^{\prime} \cup L_{i}$ so that $g_{i}\left(l_{i+1}^{\prime}\right)$ is a meridional curve of the solid torus. Then $M$ embeds in a compact 3-manifold if and only if either $M$ is simply connected at infinity and contains no infinite collection of disjoint fake 3-cells, or there $i s$ an integer $n$ such that the following three properties hold for $i>n$.

(1) $T_{i}^{\prime}$ is unknotted in $Q_{i}$.

(2) $Q_{i}$ is homeomorphic to the 3-sphere.

(3) $g_{i}\left(l_{i+1}^{\prime}\right)$ is a $\left(\gamma_{i}, 1\right)$-curve on $\partial T_{i}$ for some integer $\gamma_{i}$.

Proof. Suppose $M$ embeds in a compact 3-manifold and $M$ is not simply connected at infinity. Let $P_{i}$ denote the image in $L_{i}$ of the punctured disks given by Theorem 2.3. Thus we can apply Theorem 3.1. Part (a) implies that $P_{i+1} \cap \partial T_{i+1}^{\prime}$ is homologous to $l_{i+1}^{\prime}$ on $\partial T_{i+1}^{\prime}$. Part (c) implies that $P_{i} \cap \partial T_{i}$ is a $\left(\gamma_{i}, 1\right)$-curve on $\partial T_{i}$. Therefore property (3) of Theorem 2.3 gives property (3) of this theorem. Property (2) of Theorem 2.3 implies that $Q_{i}-\operatorname{Int}\left(T_{i}^{\prime}\right)$ is a solid torus. Now $P_{i}$ can be extended to a meridional disk $D_{i}$ of this solid torus. Part (a) of Theorem 3.1 again implies that $\partial D_{i}$ is a $(1,0)$-curve of $\partial T_{i}^{\prime}$. Therefore $Q_{i}$ is homeomorphic to the 3 -sphere and $T_{i}^{\prime}$ is unknotted in $Q_{i}$.

Conversely, suppose the three properties of this theorem hold. Then a $(1,0)$-curve on $\partial T_{i}^{\prime}$ bounds a disk $D_{i}$ in $Q_{i}-\operatorname{Int}\left(T_{i}^{\prime}\right)$. The disk $D_{i}$ can be chosen so that each component of $D_{i} \cap \partial T_{i}$ is a meridional curve on the boundary of the solid torus $Q_{i}-\operatorname{Int}\left(T_{i}^{\prime} \cup L_{i}\right)$. Now if $D_{i} \cap \partial T_{i}$ is empty for an infinite number of values of $i$, then $M$ is the union of some $M_{k}$ and a sequence of punctured 3-cells, each of which intersects the union of $M_{k}$ and the previous punctured 3-cells in a single 2-sphere boundary component. Thus $M$ is simply connected at infinity, contains no infinite collection of disjoint fake 3 -cells, and can be embedded in a compact 3-manifold. Otherwise we can apply Theorem 2.4 to complete the proof of Theorem 4.1.

The examples in this section will be constructed as follows. Let 
$L_{0}$ be the complement of the interior of an unknotted solid torus $T_{0}$ in $S^{3}$; for $i \geqq 1$, let $L_{i}$ be the complement of the interiors of two disjoint solid tori $T_{i}$ and $T_{i}^{\prime}$ in $S^{3}$. Let $g_{i}: \partial T_{i+1}^{\prime} \rightarrow \partial T_{i}$ be homeomorphisms. Then we can define the open 3-manifold $M$ as the identification space of the disjoint union of the $L_{i}$ where $x$ is identified with $g_{i}(x)$ for $x \in \partial T_{i+1}^{\prime}$. Let $K_{i}$ be the image under the identification map of $L_{i}$ in $M$.

EXAMPLE 4.2. Let $L_{i}$ be as indicated in Figure 1 . We can construct the contractible open 3-manifold of Whitehead [10] by letting $g_{i}$ be a homeomorphism that takes $l_{i+1}^{\prime}$ to $m_{i}$ and $m_{i+1}^{\prime}$ to $l_{i}$. Theorem 4.1 can be used to show that this open 3 -manifold embeds in $S^{3}$.

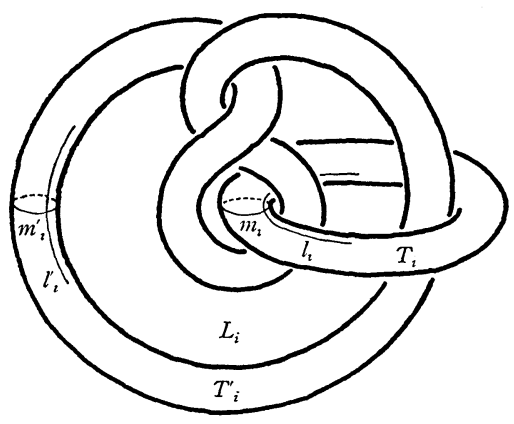

FiguRe 1

EXAMPLE 4.3. Again let $L_{i}$ be as indicated in Figure 1. We want to know what homeomorphisms $g_{i}$ will give an open 3-manifold that can be embedded in a compact 3-manifold. By Theorem 4.1 a necessary condition is that there is an integer $n$ such that for $i>n, g_{i}\left(l_{i+1}^{\prime}\right)$ is a $\left(\gamma_{i}, 1\right)$-curve on $\partial T_{i}$ for some integer $\gamma_{i}$. Since $T_{i}$ is unknotted in $S^{3}$ there is an embedding $h: T_{i}^{\prime} \cup L_{i} \rightarrow S^{3}$ of the solid torus $T_{i}^{\prime} \cup L_{i}$ onto $T_{i}^{\prime} \cup L_{i}$ in $S^{3}$ so that $h g_{i}\left(l_{i+1}^{\prime}\right)$ is a $(0,1)$ curve of $\partial T_{i}$ Thus, the compact 3 -manifold $Q_{i}$ of Theorem 4.1 is homeomorphic to $S^{3}$. But if $\gamma_{i} \neq 0$, then $h\left(T_{i}^{\prime}\right)$ is a regular neighborhood of a nontrivial twist knot. See [11] or [1] for the relevant facts about twist knots. Thus condition (1) of Theorem 4.1 is not met unless $\gamma_{i}=0$.

REMARK. Suppose for each $i \geqq 0$ that $g_{i}\left(l_{i+1}^{\prime}\right)$ is a $\left(\gamma_{i}, 1\right)$-curve on $\partial T_{i}$ for any integer $\gamma_{i}$. If we choose $g_{i}\left(m_{i+1}^{\prime}\right)=l_{i}$ for each $i \geqq 0$, then $M$ will be monotone union of solid tori. Since each solid torus will be contractible in the succeeding one, $M$ will also be contractible.

EXAMPle 4.4. Let $L_{i}$ be as indicated in Figure 2. Notice that 
there is a punctured disk in $L_{i}$ bounded by a $(1,0)$-curve on $\partial T_{i}^{\prime}$ and two $(1,0)$-curves on $\partial T_{i}$. This punctured disk can be visualized in Figure 2 as the union of an annulus with boundary components $a_{1} \cup b_{1}$ and $d_{1}$, a disk with boundary $b_{1} \cup c_{1} \cup b_{2} \cup c_{2}$, and an annulus with boundary components $a_{2} \cup b_{2}$ and $d_{2}$.

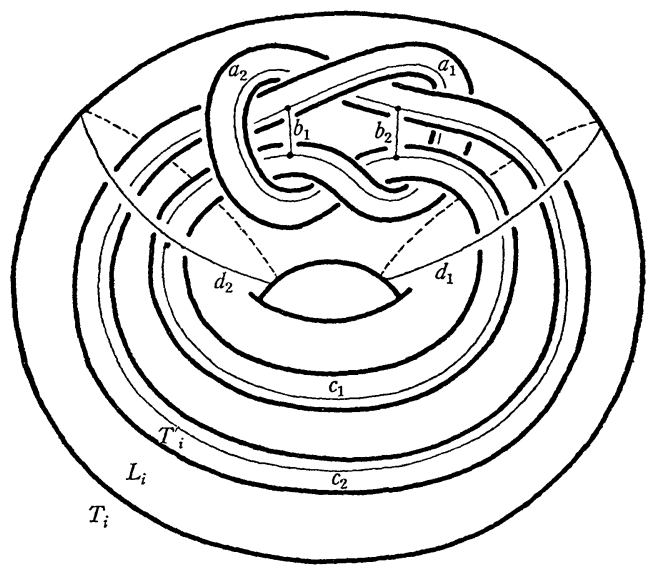

FIGURE 2

Nevertheless, if $g_{i}\left(l_{i+1}^{\prime}\right)$ is a $(1,0)$-curve on $\partial T_{i}, Q_{i}$ will be homeomorphic to $S^{2} \times S^{1}$. In fact, in order for $Q_{i}$ to be a 3-sphere, $g_{i}\left(l_{i+1}^{\prime}\right)$ must be a $\left(\gamma_{i}, 1\right)$-curve on $\partial T_{i}$. As in the previous example, we can reembed $T_{i}^{\prime} \cup L_{i}$ so that the image of $g_{i}\left(l_{i+1}^{\prime}\right)$ is a meridional curve of the complementary solid torus. Figure 3 is a typical picture of the knotted torus $T_{i}^{\prime}$ in the 3-sphere $Q_{i}$.

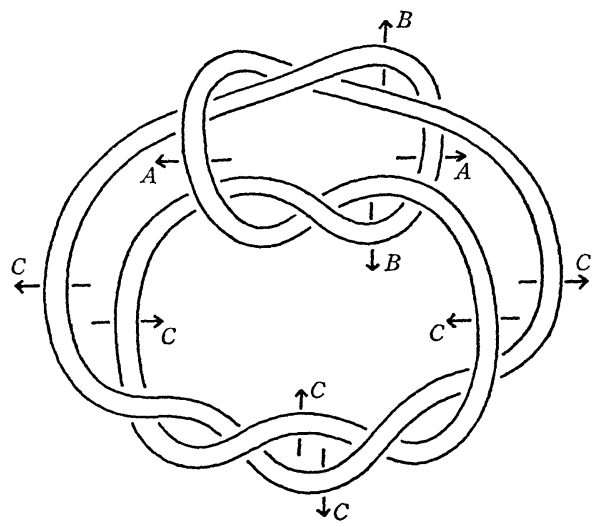

FIGURE 3

An epimorphism from the fundamental group of the complement of $T_{i}^{\prime}$ onto the trefoil knot group can be obtained as follows. Compute 
the Wirtinger presentation [2] for the group of the complement of $T_{i}^{\prime}$. Then identify all generators that have the same label in Figure 3. The resulting group will be a presentation of the trefoil knot group:

$$
\left\langle A, B, C \mid A C A^{-1} B^{-1}, B A B^{-1} C^{-1}, C B C^{-1} A^{-1}\right\rangle \text {. }
$$

Therefore $T_{i}^{\prime}$ is a nontrivial knot, and so it does not satisfy condition (1) of Theorem 4.1.

Notice that $\partial L_{i}$ is incompressible in $L_{i}$. Indeed $\partial T_{i}^{\prime}$ is incompressible in $L_{i} \cup T_{i}$ since $T_{i}^{\prime}$ is knotted in $S^{3}$. Also a meridional disk of $T_{i}^{\prime} \cup L_{i}$ that is disjoint from $T_{i}^{\prime}$ could be used together with the punctured disk in $L_{i}$ to contradict the fact that $T_{i}^{\prime}$ is knotted. Thus $M$ is not simply connected at infinity.

It follows that there are no sewing maps $g_{i}$ for which the resulting open 3-manifold $M$ will embed in a compact 3-manifold. In fact it follows from Theorem 2.3 that if there is an infinite number of values of $i$ for which $K_{i}$ is homeomorphic to such an $L_{i}$ with $\partial M_{i}$ corresponding to $\partial T_{i}$, then the open 3-manifold cannot be embedded in a compact 3-manifold.

THEOREM 4.5. Suppose each $q_{i}=1$. Suppose $T_{i}$ is unknotted in $S^{3}, T_{i}^{\prime}$ is knotted in $S^{3}$, and $T_{i}^{\prime}$ has wrapping number one in the solid torus $S^{3}-\operatorname{Int}\left(T_{i}\right)$. Then $M$ can be embedded in a compact 3-manifold if and only if there is an integer $n$ so that $g_{i}\left(m_{i+1}^{\prime}\right)$ is $a(1,0)$-curve on $\partial T_{i}$ for all $i>n$.

Proof. Suppose $M$ can be embedded in a compact 3-manifold. Using the fact that $\partial T_{i}^{\prime}$ and $\partial T_{i}$ are incompressible in $L_{i}$, it follows that $M$ is not simply connected at infinity. Let $P_{i}$ denote the images in $L_{i}$ of the punctured disks given by Theorem 2.3.

Notice that $P_{i} \cap \partial T_{i}^{\prime}$ is a $(0,1)$-curve on $\partial T_{i}^{\prime}$. For suppose it is an $(\alpha, \beta)$-curve with $\alpha \neq 0$. Then there is a 3-cell in $S^{3}-T_{i}$ that intersects $L_{i}$ in a cube with a knotted hole $C$. We can modify $P_{i}$ so that $P_{i} \cap C \cap \partial T_{i}^{\prime}$ consists of exactly $\alpha$ arcs. We can further modify $P_{i}$ so that each component of $P_{i} \cap \partial C$ is nontrivial on $\partial C$. Then each component of $P_{i} \cap C$ is a compressing disk for $\partial C$ in $C$. This contradicts the hypothesis that $T_{i}^{\prime}$ is knotted. For more details see $\S 5$ of [4].

By part (f) of Theorem 3.1 each component of $P_{i} \cap \partial T_{i}$ is a $(1,0)$ curve on $\partial T_{i}$. The result of the previous paragraph applied to $P_{i+1}$ in $L_{i+1}$ and property (3) of Theorem $2.3 \mathrm{imply}$ that $g_{i}\left(m_{i+1}^{\prime}\right)$ is a $(1,0)$ curve on $\partial T_{i}$.

Suppose conversely that there is an integer $n$ so that $g_{i}\left(m_{i+1}^{\prime}\right)$ is a $(1,0)$-curve on $\partial T_{i}$ for all $i>n$. Since $T_{i}^{\prime}$ has wrapping number 
one in $S^{3}-\operatorname{Int}\left(T_{i}\right)$, there is an annulus $P_{i}$ in $L_{i}$ bounded by the $(0,1)$-curve $m_{i}^{\prime}$ on $\partial T_{i}^{\prime}$ and the $(1,0)$-curve $l_{i}$ on $\partial T_{i}$.

Let $Q_{i}$ be the 3-manifold obtained by attaching a solid torus to $T_{i}^{\prime} \cup L_{i}$ along $\partial T_{i}$ so that $l_{i}$ is a meridional curve of the solid torus. Notice that $Q_{i}$ is homeomorphic to $S^{2} \times S^{1}$. To apply Theorem 2.4 it suffices now to show that $Q_{2}-\operatorname{Int}\left(T_{i}^{\prime}\right)$ is a solid torus. We can extend $P_{i}$ to a disk $D_{i}$ in $Q_{i}-\operatorname{Int}\left(T_{i}^{\prime}\right)$ by attaching a meridional disk of the solid torus $Q_{i}-\operatorname{Int}\left(T_{i}^{\prime} \cup L_{\imath}\right)$ to $P_{i} \cap \partial T_{i}$. Now $D_{i}$ can be extended to a nonseparating 2-sphere in $Q_{i}$ by attaching a meridional disk of $T_{i}^{\prime}$ to $\partial D_{i}$. Since $Q_{i}$ is a prime 3 -manifold, cutting $Q_{i}-\operatorname{Int}\left(T_{i}^{\prime}\right)$ along $D_{\imath}$ yields a 3 -cell. Thus $Q_{i}-\operatorname{Int}\left(T_{i}^{\prime}\right)$ is a solid torus.

ExAMPLE 4.6. Let $L_{i}$ be as indicated in Figure 4. This illustrates the situation described in Theorem 4.5.

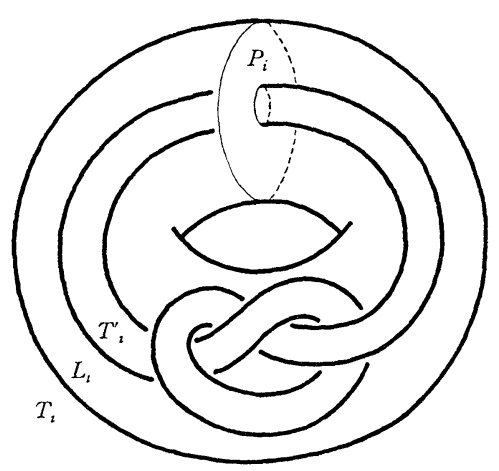

Figure 4

If $g_{i}\left(m_{i+1}^{\prime}\right)$ is a $(1,0)$-curve on $\partial T_{i}$ for all $i \geqq 0$ then the resulting open 3-manifold $M$ is one of the open 3-manifolds given by Tucker in Example 3 of [9]. Even though $M$ does not embed in any compact, irreducible 3-manifold, it does embed in $S^{2} \times S^{1}$. To see this notice that there is a homeomorphism of $L_{i}$ onto itself that interchanges the two boundary components. Indeed, cut $L_{i}$ along the annulus $P_{i}$ and let $L_{i}^{*}$ denote the resulting cube with a knotted hole. There is an isotopy of $L_{i}^{*}$ that interchanges $L_{i}^{*} \cap \partial T_{i}$ and $L_{i}^{*} \cap \partial T_{i}^{\prime}$. This can be used to define the desired homeomorphism of $L_{i}$. It now follows that $M-\operatorname{Int}\left(M_{0}\right)$ can be embedded in a solid torus as the complement of the intersection of a decreasing sequence of solid tori each embedded in the previous one as $T_{i}^{\prime}$ is embedded in $S^{3}-\operatorname{Int}\left(T_{i}\right)$ in Figure 4. 


\section{REFERENCES}

1. R. H. Bing and J. M. Martin, Cubes with knotted holes, Trans. Amer. Math. Soc., 115 (1971), 217-231.

2. Richard H. Crowell and Ralph H. Fox, Introduction to Knot Theory, Ginn and Company, Boston, 1963.

3. C. H. Edwards, Jr., Open 3-manifolds which are simply connected at infinity, Proc. Amer. Math. Soc., 14 (1963), 391-395.

4. Wolfgang Haken, Some results on surfaces 3-manifolds, Studies in Modern Topology, Math. Assoc. Amer. (distributed by Prentice-Hall, Englewood Cliffs, N. J.), 1968, 39-98. 5. J. M. Kister and D. R. McMillan, Jr., Locally Euclidean factors of $E^{4}$ which cannot be embedded in $E^{3}$, Ann. Math., (2) 76 (1962), 541-546.

6. H. Kneser, Geschlossene Flächen in dreidimensionalen Mannigfaltigkeiten, Jber. Deutsch. Math.-Verein., 38 (1929), 248-260.

7. Tom Knoblauch, Imbedding deleted 3-manifold neighborhoods in $E^{3}$, Illinois J. Math., 18 (1974), 598-601.

8. D. R. McMillan, Jr., Some contractible open 3-manifolds, Trans. Amer. Math. Soc., 102 (1962), 373-382.

9. T. W. Tucker, Non-compact 3-manifolds and the missing-boundary problem, Topology, 13 (1974), 267-273.

10. J. H. C. Whitehead, A certain open manifold whose group is unity, Quart. J. Math., (Oxford) 6 (1935), 268-279.

11. — On doubled knots, J. London Math. Soc., 12 (1937), 63-71.

12. Alden Wright, Mappings from 3-manifolds onto 3-manifolds, Trans. Amer. Math. Soc., 167 (1972), 479-495.

Received November 3, 1977. The second author was partially supported by Western Michigan University Faculty Research Fund.

VANDERBILT UNIVERSITY

NASHVILLE, TN 37235

AND

WeSTERN Michigan UNIVERSITY

KaLAMAZOO, MI 49008 



\section{PACIFIC JOURNAL OF MATHEMATICS}

\section{EDITORS}

DoNALD BABBITT (Managing Editor)

University of California

Los Angeles, California 90024

HUGo Rossi

University of Utah

Salt Lake City, UT 84112

C. C. MOORE and ANDREW OGG

University of California

Berkeley, CA 94720
J. DUGUNDJI

Department of Mathematics University of Southern Californı Los Angeles, California 90007

R. Finn and J. Milgram Stanford University Stanford, California 94305

\section{ASSOCIATE EDITORS}

E. F. BECKENBACH

B. H. Neumann
F. WOLF

K. YOSHIDA

\section{SUPPORTING INSTITUTIONS}

UNIVERSITY OF BRITISH COLUMBIA CALIFORNIA INSTITUTE OF TECHNOLOGY UNIVERSITY OF CALIFORNIA MONTANA STATE UNIVERSITY UNIVERSITY OF NEVADA, RENO NEW MEXICO STATE UNIVERSITY OREGON STATE UNIVERSITY UNIVERSITY OF OREGON
UNIVERSITY OF SOUTHERN CALIFORNIA STANFORD UNIVERSITY UNIVERSITY OF HAWAII UNIVERSITY OF TOKYO UNIVERSITY OF UTAH WASHINGTON STATE UNIVERSITY UNIVERSITY OF WASHINGTON 


\section{Pacific Journal of Mathematics}

Vol. 82, No. $1 \quad$ January, 1979

Werner Bäni, Subspaces of positive definite inner product spaces of countable dimension ...................................... 1

Marilyn Breen, The dimension of the kernel of a planar set..............

Kenneth Alfred Byrd, Right self-injective rings whose essential right ideals

are two-sided

Patrick Cousot and Radhia Cousot, Constructive versions of Tarski's fixed

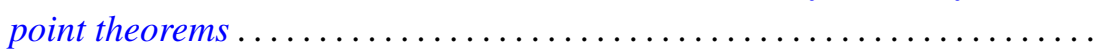

Ralph S. Freese, William A. Lampe and Walter Fuller Taylor, Congruence lattices of algebras of fixed similarity type. $I \ldots \ldots \ldots \ldots \ldots \ldots \ldots$

Cameron Gordon and Richard A. Litherland, On a theorem of Murasugi .....

Mauricio A. Gutiérrez, Concordance and homotopy. I. Fundamental

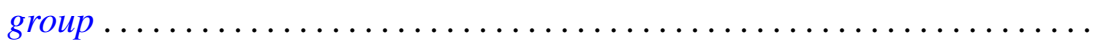

Richard I. Hartley, Metabelian representations of knot groups .............

Ted Hurley, Intersections of terms of polycentral series of free groups and free

Lie algebras ........................................

Roy Andrew Johnson, Some relationships between measures ............ 117

Oldřich Kowalski, On unitary automorphisms of solvable Lie algebras .......

Kee Yuen Lam, $K O$-equivalences and existence of nonsingular bilinear

maps...................................................

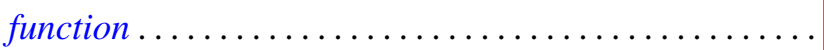

Robert A. Messer and Alden H. Wright, Embedding open 3-manifolds in compact 3-manifolds ............................

Gerald Ira Myerson, A combinatorial problem in finite fields. I . .

James Nelson, Jr. and Mohan S. Putcha, Word equations in a band of paths.

Baburao Govindrao Pachpatte and S. M. Singare, Discrete generalized Gronwall inequalities in three independent variables . .

William Lindall Paschke and Norberto Salinas, $C^{*}$-algebras associated with free products of groups ........................

Bruce Reznick, Banach spaces with polynomial norms ....

David Rusin, What is the probability that two elements of a finite group

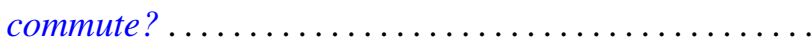

M. Shafii-Mousavi and Zbigniew Zielezny, On hypoelliptic differential operators of constant strength ...

Joseph Gail Stampfli, On selfadjoint derivation ranges .... . . .

Robert Charles Thompson, The case of equality in the matrix-valued triangle

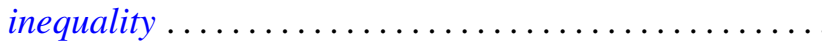

Marie Angela Vitulli, The obstruction of the formal moduli space in the negatively graded case. 